\title{
Long-term conceptual implicit memory: A decade of evidence
}

\author{
David R. Thomson and Bruce Milliken \\ McMaster University, Hamilton, Ontario, Canada \\ AND \\ DANiel SMILEK \\ University of Waterloo, Waterloo, Ontario, Canada
}

\begin{abstract}
Demonstrations of long-term implicit memory are numerous, but to date they have been reported in what might be thought of as perceptually driven tasks. In the present experiment, a low-frequency U.S. state name was presented verbally to participants within the context of a memory-course lecture, and the influence of that experience was measured indirectly 4 to 8 weeks later using a state-name-generation task. Participants were significantly more likely to generate the critical state name when it had been presented in an earlier lecture than when it had not been presented in an earlier lecture, a novel demonstration of long-term, conceptually driven priming after a single stimulus exposure.
\end{abstract}

This article presents the results of a classroom demonstration that was carried out over the course of 10 years and that has reliably produced memory effects that hold over long delays. We describe this effect here because it appears to hold a unique place in the memory literature; that is, it constitutes a unique demonstration of long-term, implicit, conceptually driven memory.

The prominence in the memory literature of the distinction between implicit and explicit memory stems from a host of well-known dissociations involving tasks that either do or do not require conscious access to a particular encoding experience (see, e.g., Jacoby \& Dallas, 1981; Tulving, Schacter, \& Stark, 1982). For example, Tulving et al. tested participants in both recognition and fragmentcompletion tasks following retention intervals of $1 \mathrm{~h}$ or 7 days. Performance in the explicit recognition memory task was much worse for the longer retention interval than for the shorter retention interval, whereas priming effects in the presumably implicit fragment-completion task were unaffected by retention interval. Such a result might therefore be argued to demonstrate that separate memory systems underlie performance on implicit- and explicit-memory tasks.

Whereas some researchers have cited such task dissociations as support for separate memory systems (for a review, see Squire, 2004), others have noted that implicit and explicit tasks often differ in the type of processing that they require (Blaxton, 1989; Roediger, 1990; Roediger \& McDermott, 1995). To appreciate this alternativeprocessing perspective, consider that the recognition memory task could be considered both explicit and con- ceptually driven, in the sense that recognizing often involves the recapitulation of meaning-based encoding processes that occurred during the study phase. In contrast, the fragment-completion task can be argued to be both implicit and perceptually driven, in that perceptual processing during the test phase leads to some of the same perceptual operations that are performed during encoding of items in the study phase. From a processing perspective, it is this recapitulation of perceptual operations, at least in part, that enhances performance for old items relative to new items. In line with this alternative processing perspective, Blaxton reported a study demonstrating that it may be the perceptual-versus-conceptual processing requirements of a task, rather than the implicit-versusexplicit status of a task, that predicts task dissociations.

Although the interpretation of task dissociations remains controversial, a consequence of this theoretical debate is that tasks are now commonly classified both in terms of their explicit or implicit requirement to remember and in terms of the perceptual or conceptual nature of the cues that are driving the critical retrieval processes. Within this broad task-classification scheme, there are many examples of long-term, implicit, perceptually driven memory effects (Cave, 1997; Kolers, 1976; Mitchell, 2006; Tulving et al., 1982), whereas long-term, implicit, conceptually driven memory effects are conspicuously absent from the literature. Although Zeelenberg and Pecher (2003) reported a conceptual cross-language priming effect in which there were an average of 65 intervening items between study and test for a particular target item, the time delay between study and test (not reported) appears to have been

D. R.Thomson, thomsodr@mcmaster.ca 
on the order of minutes. To our knowledge, a longer term effect - on the order of days, weeks, or more - has never been reported, which could easily lead one to conclude that long-term implicit memory effects are a consequence of perceptual learning exclusively.

The present study measured conceptually driven priming effects using a procedure in which participants were presented with target items (U.S. state names) in the context of a verbal lecture, and the likelihood of their generating these target items was tested indirectly 4 to 8 weeks later. A demonstration of long-term, implicit, conceptually driven memory in this context would be noteworthy, since it would undermine the view that longterm implicit memory effects are entirely the product of perceptual learning.

\section{METHOD}

\section{Participants}

All participants were undergraduates at McMaster University who were enrolled in a 3rd-year memory course between 1996 and $2007(N=245)$. The students took part in the experiment as part of a yearly in-class demonstration.

\section{Materials}

The critical state names that were used in this experiment were five U.S. state names that were selected for their particularly low probability of generation by Canadian students: West Virginia, Wisconsin, Wyoming, Delaware, and Rhode Island.

\section{Procedure}

Each year from 1996 to 2007, students who were enrolled in a 3rdyear course on human memory attended a lecture on retrieval strategies. At some point in the lecture, the critical U.S. state name was presented in the context of a discussion of the distinction between the state name being unavailable versus inaccessible, as a result of the absence of an appropriate retrieval strategy. The demonstration typically proceeded as follows: "When trying to recall all 50 U.S. states, you might employ an alphabetic retrieval strategy to boost your performance, so that when, for instance, you come to the letter 'D,' you'll have some chance of remembering Delaware." This part of the lecture, in which the critical state name was embedded, constituted the study session of the experiment. Encoding can be described as incidental, in that students were unaware that they would later be asked to generate state names. For the first experimental session, Wyoming was chosen arbitrarily as the critical state name. When these data were collected, the least frequently reported state for that year was used as the critical state name the following year. This strategy yielded five critical state names across five years. Each state name was used once more, resulting in 10 experimental sessions in total, although an experimenter error in 2005 (ironically, a memory error as to which critical state name was actually presented at study) resulted in the exclusion of those data and the running of an additional session in 2007.
The test phase of the experiment occurred in a subsequent lecture each year, 4 to 8 weeks after the initial study session. Participants were asked to write on a sheet of paper as many of the 50 states as they could remember in $10 \mathrm{~min}$.

\section{RESULTS}

For each experimental session, the state names were rank-ordered from most to least frequently generated. The critical state name that was mentioned during the study phase was labeled biased; the four critical state names that were not mentioned in a study phase were labeled unbiased. Across the 10 experimental sessions, therefore, two biased rank orders and eight unbiased rank orders were obtained for each critical state name. These rank orders are presented in Table 1. Collapsing the ranks across the 2 critical years and the 8 additional years yielded a single mean biased rank and a single mean unbiased rank for each of the five critical states, which are displayed in Figure 1.

The mean difference in rank from unbiased to biased for the five critical state names was $16.3 ; 95 \%$ confidence intervals that were obtained via nonparametric bootstrap analysis, per Efron and Tibshirani (1993), were $21.50<$ $\mu<31.62$ and $40.65<\mu<44.84$ for the biased and unbiased ranks, respectively. We thus concluded that the mean biased and mean unbiased ranks came from different distributions.

Awareness of the source of this bias effect was measured informally each year. Participants were presented with the rank-ordered data that had been obtained at the time of test, and they were asked first to speculate on whether there were any state names that were ranked higher than they might expect. Participants gave a host of answers to this question, which included only rarely the biased state name. The instructor then pointed out that he found the biased state name to be ranked unusually high, and he asked the class whether they could think of any reasons why that state was ranked as high as it was. In some of the test sessions, a single student mentioned that the biased state name had been mentioned in a prior lecture; in others, no students did so. In cases in which the biased state name was mentioned by a student, the suggestion appeared to be made with relatively low confidence, more as a question to the instructor than as a statement. In these cases, the instructor then asked the class whether they remembered the biased state name having been presented. In many cases, no other students reported remembering the initial study encounter, and in the remaining cases, no more than 1 or 2 additional students gave an indication that they remem-

Table 1

Mean Rank Order of Critical State Names As a Function of Year

\begin{tabular}{lllllllllll}
\hline \multicolumn{1}{c}{ State } & 1996 & 1997 & 1998 & 1999 & 2000 & 2002 & 2003 & 2004 & 2006 & 2007 \\
\hline Wyoming & $\mathbf{1 1}$ & 43 & 49.5 & 46.5 & 46 & $\mathbf{2 1}$ & 50 & 47.5 & 40.5 & 30.5 \\
West Virginia & 50 & $\mathbf{3 2}$ & 43.5 & 46.5 & 46 & 46 & $\mathbf{2 1}$ & 44.5 & 47 & 50 \\
Wisconsin & 48.5 & 49.5 & $\mathbf{1 7 . 5}$ & 34.5 & 39.5 & 25 & 40 & 41.5 & 44 & $\mathbf{3 6}$ \\
Delaware & 48.5 & 47.5 & 50 & $\mathbf{4 0 . 5}$ & 48.5 & 46 & 40 & $\mathbf{2 4 . 5}$ & 47 & 47.5 \\
Rhode Island & 44 & 40 & 36 & 34.5 & $\mathbf{2 7}$ & 35 & 47 & 28 & $\mathbf{3 5}$ & 26 \\
\hline
\end{tabular}

Note-Values in bold represent rank orders for state names that were biased. 


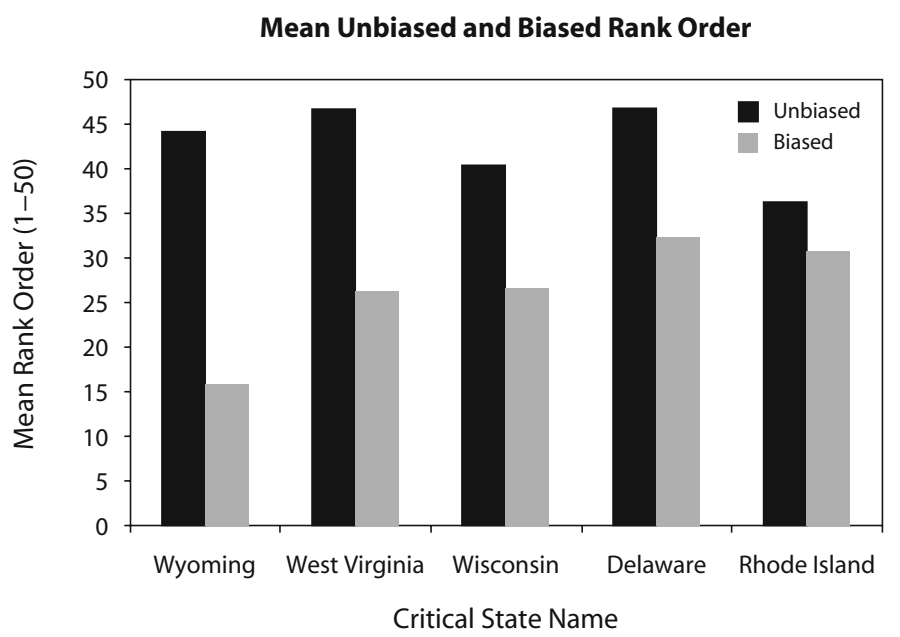

Figure 1. The mean biased and unbiased rank $(1=$ most frequently reported, 50 = least frequently reported) for each of the five target state names is shown, collapsed across all experimental sessions.

bered the initial study encounter. Only with a great deal of additional contextual support (e.g., other details of the lecture) were students slowly convinced that the target state name had been mentioned in a prior lecture. Although this method of inquiry is somewhat informal, the behavior of the class gave a very clear impression that deliberate recruitment of prior learning episodes was highly unlikely to have been the source of the large bias effect in rank for the critical state names.

\section{DISCUSSION}

The results of the present study demonstrate that generation of low-frequency category exemplars can be influenced by a single mention of one of those exemplars many weeks earlier; moreover, this effect appears to occur alongside an absence of explicit recollection of the initial encoding episode. To our knowledge, this long-term, implicit, conceptually driven memory effect is unique in the literature.

We believe that the classroom demonstration is sufficiently compelling to stand on its own, yet our evidence concerning the awareness status of participants hinged on participants' self-report, and our claim that the result constituted a unique form of implicit memory requires some form of defense of those methods. To that end, we note that the implicit-explicit distinction relates to states of subjective experience, and self-report therefore seems a reasonable and straightforward method of measuring those subjective states. Although such methods might well have been considered unscientific several decades ago, an increasing number of researchers are making use of subjective report to study conscious experience (e.g., Mitchell, 2006, whose use of self-report in a study of long-term priming was similar to ours). Indeed, Merikle, Smilek, and Eastwood (2001) have argued that self-report of what an individual has perceived is often just as reli- able as more objective measures of perception. An additional concern is that the informality of the method that was used to measure self-reported awareness may have resulted in something less than an exhaustive measure of participants' awareness. There is no way to be sure that we exhaustively tapped participants' awareness of the original encoding episode, but the compelling nature of the demonstration stems in large part from the obvious effort by the experimenter/lecturer to encourage students to offer reasonable hypotheses for the relatively high rank of the biased state name. We made this attempt to elicit conscious report of the original encoding episode as salient an aspect of the method as possible in order to ensure that in later debriefing the participants would appreciate that we had indeed attempted to provide as exhaustive a measure of conscious experience as possible. Across 10 years of the study, many former students have remarked that the demonstration was compelling, and we presume that our overstated attempts to elicit conscious report of the original encoding experience, to little effect, contributed to this impression.

We thus propose that this long-term conceptually driven memory effect indeed occurs in the absence of awareness, and that it therefore holds a unique place in the memory literature. It is worth noting, however, that one other recent study has addressed at least some of the issues that were raised by our study. Hunt and Lamb (2006) presented participants with sentences that contained a category followed by a low-frequency exemplar of that category (categoryexemplar) or with sentences that contained a low-frequency exemplar followed by its category (exemplar-category). Participants were presented with category labels at test and were asked to report the first eight instances of each category that came to mind. The category-exemplar items produced greater priming under conditions of divided attention than did the exemplar-category items. The authors claimed that the key to observing reliable priming of low-frequency 
items under divided attention with a single stimulus exposure is to present the category in a way that biases comprehension toward low-frequency instances. It is unlikely that a general biasing toward low-frequency instances occurred in our experiment, since the observed result did not generalize across all low-frequency state names in a given session but instead was limited to the particular state name that was mentioned at study. Nonetheless, the results of the Hunt and Lamb study do demonstrate robust biasing of low-frequency category exemplars with a single presentation, and the study did employ a category-production task that was similar to ours. As these authors noted, the absence of any perceptual features of category exemplars in the task instructions at the time of test, together with the requirement to generate category exemplars rather than to remember previously presented category exemplars, made theirs an implicit (or indirect) conceptually driven memory test. Our memory test had these same properties and therefore very likely tapped into similar processes. Additionally, the effect that we report here survived a lag between study and test of at least 1 month, which constitutes its unique contribution relative to the study of Hunt and Lamb.

We noted at the start of this article that long-term, implicit, conceptually driven memory effects are conspicuous in their absence from the literature, particularly given the attention that researchers have paid to the implicitexplicit distinction over the past 25 or so years. We want to make clear again that the presence of such an effect in this study does not tip the balance in favor of either separatesystems or more processing-oriented interpretations of dissociations between implicit- and explicit-memory test performance. We believe that the present results do constrain the interpretation of at least some of the dissociations that have shaped this debate, however, in particular those involving retention-interval effects.

For example, processing-oriented accounts of the Tulving et al. (1982) study that are described above might offer that retention interval has its effect primarily on conceptually related processes. According to this view, the dissociation in the Tulving et al. study owes to different effects of retention interval on the products of conceptual and perceptual encoding processes, rather than to different effects of retention interval on implicit- and explicitmemory systems. Our point is that the conspicuous absence of any long-term, implicit, conceptually driven memory effect in the literature might be seen as support for this particular processing interpretation. The present results highlight that the products of conceptually related encoding processes can endure a substantial retentioninterval manipulation, so it is much less clear that one can, a priori, identify the processes that are affected by retention interval as being conceptual rather than perceptual and therefore explain away a dissociation by reference to the selective effect of retention interval on conceptual processes. On the other hand, a multiple-systems interpretation of dissociations might well appear to be strengthened by the idea that different learning systems have different learning constraints, and learning within modular perception systems ought to be more robust to manipulations of retention interval than is higher order learning that imparts meaning to perceptual learning that is done elsewhere. Yet the presence of long-term, implicit, conceptually driven memory effects undermines the idea that dissociations involving retention interval necessarily reflect the different constraints on learning within separate systems. In summary, although we do not pretend that the data that are reported here favor either a separate-systems or processing interpretation of implicit- or explicit-memory task dissociations, we do think that the long-term, implicit, conceptually driven memory effect that we report here constrains how proponents of both theoretical camps incorporate some well-known dissociations involving manipulations of retention interval.

Finally, we note that there may well be opportunities for proponents of processing-oriented frameworks, such as transfer-appropriate processing (Morris, Bransford, \& Franks, 1977), to extend the present research in some interesting directions. In particular, in the absence of perceptual overlap between study and test in the present experiment, the key to understanding why the effect persists over a retention interval of 1 month or more may lie in identifying potential sources of conceptual-processing overlap between study and test. One possible source of conceptual-processing overlap relates to the fact that participants were exposed to the critical state name in the context of the use of an alphabetic strategy to recall state names. Informal reports from participants at the time of debriefing suggest that many of them did rely on an alphabetic strategy to perform the recall task. Another possible source of conceptual-processing overlap is suggested by research showing that individuals can be primed to form specific goals when perceiving and encoding information during a study period and that when those goals are activated at test, information that was previously linked to these goals will be better remembered (Bargh, Gollwitzer, Lee-Chai, Barndollar, \& Trötschel, 2001; Chartrand \& Bargh, 1996).

Clearly, additional research is required to determine whether the aforementioned sources of conceptual overlap at encoding and retrieval are critical to the observed effect, but future studies are also needed to determine the generality of the effect that we report here. We have described our research as if it represents a larger category of methods in which biasing of low-frequency category exemplars is tested using a category-cued production task, yet it remains possible that the generation of U.S. state names by Canadians has some idiosyncratic properties that would make replication outside this literal task context challenging. We hope that the report of this classroom demonstration invites others to extend the research to other tasks, and that the challenge ultimately proves to be a modest one.

\section{AUTHOR NOTE}

This research was supported and funded by an NSERC Discovery Grant. We thank the many students enrolled in Psychology 3VV3 for their helpful comments over the years during which the study was conducted. Additionally, we thank Ellen Maclellan for help with data coding, as well as Henry Roediger III and two anonymous reviewers for their comments on the manuscript. Correspondence concerning this article 
should be addressed to D. R. Thomson, Department of Psychology, Neuroscience and Behaviour, McMaster University, Hamilton, ON, L8S 4K1 Canada (e-mail: thomsodr@mcmaster.ca).

\section{REFERENCES}

Bargh, J. A., Gollwitzer, P. M., Lee-Chai, A., Barndollar, K., \& TRÖTSCHEL, R. (2001). The automated will: Nonconscious activation and pursuit of behavioral goals. Journal of Personality \& Social Psychology, 81, 1014-1027.

BlaXton, T. A. (1989). Investigating dissociations among memory measures: Support for a transfer-appropriate processing framework. Journal of Experimental Psychology: Learning, Memory, \& Cognition, 15, 657-668.

CAVE, C. B. (1997). Very long-lasting priming in picture naming. Psychological Science, 8, 322-325.

Chartrand, T. L., \& Bargh, J. A. (1996). Automatic activation of impression formation and memorization goals: Nonconscious goal priming reproduces effects of explicit task instructions. Journal of Personality \& Social Psychology, 71, 464-478.

Efron, B., \& TibShirani, R. J. (1993). An introduction to the bootstrap. London: Chapman \& Hall.

Hunt, R. R., \& LAMB, C. A. (2006). What does it take to implicitly prime low-frequency category exemplars? Journal of Experimental Psychology: Learning, Memory, \& Cognition, 32, 249-258.

JACOBY, L. L., \& DALlaS, M. (1981). On the relationship between autobiographical memory and perceptual learning. Journal of Experimental Psychology: General, 110, 306-340.
Kolers, P. A. (1976). Pattern-analyzing memory. Science, 191, 12801281.

Merikle, P. M., Smilek, D., \& Eastwood, J. D. (2001). Perception without awareness: Perspectives from cognitive psychology. Cognition, 79, 115-134.

Mitchell, D. B. (2006). Nonconscious priming after 17 years: Invulnerable implicit memory? Psychological Science, 17, 925-929.

Morris, C. D., Bransford, J. D., \& Franks. J. J. (1977). Levels of processing versus transfer appropriate processing. Journal of Verbal Learning \& Verbal Behavior, 16, 519-533.

RoEDiger, H. L., III (1990). Implicit memory: Retention without remembering. American Psychologist, 45, 1043-1056.

RoEdiger, H. L., III, \& MCDERMOTT, K. B. (1995). Creating false memories: Remembering words not presented in lists. Journal of Experimental Psychology: Learning, Memory, \& Cognition, 21, 803-814.

SQuire, L. R. (2004). Memory systems of the brain: A brief history and current perspective. Neurobiology of Learning \& Memory, 82, 171-177.

Tulving, E., Schacter, D. L., \& Stark, H. A. (1982). Priming effects in word-fragment completion are independent of recognition memory. Journal of Experimental Psychology: Learning, Memory, \& Cognition, 8, 336-342.

Zeelenberg, R., \& Pecher, D. (2003). Evidence for long-term crosslanguage repetition priming in conceptual implicit memory tasks. Journal of Memory \& Language, 49, 80-94.

(Manuscript received March 5, 2009; revision accepted for publication April 9, 2009.) 